\title{
ANÁLISIS DE EXPOSICIONES ARTÍSTICAS TEMPORALES EN LA COMUNIDAD AUTÓNOMA DE CATALUÑA. 2010- 2015
}

\section{ANALYSIS OF TEMPORARY ARTISTIC EXHIBITIONS IN THE AUTONOMOUS COMMUNITY OF CATALONIA. 2010- 2015}

\author{
Beatriz Parejo Ramos \\ (Historia del Arte, Universidad de Málaga, España) \\ artismusas@gmail.com
}

Recibido el 06 de septiembre de 2017; aceptado el 20 de noviembre de 2017

\begin{abstract}
RESUMEN: El proyecto de investigación Exhibitium, llevado a cabo por el grupo de investigación iArtHis_Lab de la Universidad de Málaga, ha desarrollado un repositorio de datos con las exposiciones artísticas que han tenido lugar en España desde 2010 a 2015. En este marco, mi trabajo se ha basado en el análisis de las exposiciones realizadas en la comunidad autónoma de Cataluña, análisis que se ha llevado a cabo aplicando estrategias basadas en el procesamiento de datos masivos, como análisis de redes o procesos de clustering. Los resultados me han permitido responder ciertas preguntas que aportan información sobre el escenario expositivo de esta región de acuerdo con determinados aspectos.
\end{abstract}

PALABRAS CLAVE: Exposiciones artísticas temporales, Historia del Arte Digital, Humanidades Digitales, Cataluña, análisis de datos, análisis de redes.

ABSTRACT: This work falls within the Digital Humanities' research projects. An introduction to this field is given in the first part of the work, along with a general glimpse to the computational- based studies in the Humanities and particularly in Art History. Afterward, the Exhibitium Project, developed at the University of Málaga, is explained. According to its objectives, a data repository about art exhibitions held in Spain from 2010 to 2015 has been built within the framework of this project. This is the data repository that I have used in my research, which has focused on the analysis of the art exhibitions held in the Cataluña region. Diverse data-driven analysis techniques, such as network analysis of clustering process have 
been applied. The results obtained have allow me to respond certain questions, which, in turn, have make possible to describe the art exhibition scenario of this region on the basis of specific features.

KEYWORDS: Temporary art exhibitions, Digital Art History, Digital Humanities, Cataluña, data- driven analysis, network analysis.

\section{INTRODUCCIÓN}

El objetivo de este artículo es mostrar el análisis de las exposiciones artísticas desarrolladas en Cataluña durante los años 2010-2015, utilizando para ello estrategias de análisis de grandes conjuntos de datos. De esta forma, a través del estudio se responderá a determinados interrogantes planteados en relación a los movimientos exhibidos, los artistas participantes, las redes que se crean entre ellos, la nacionalidad, el género, etc.

El análisis de las exposiciones se realiza a partir de datos indexados anteriormente en ExpoFinder, infraestructura tecnológica que pertenece al proyecto Exhibitium 1, coordinado por el grupo de investigación iArtHis_Lab del Departamento de Historia del Arte de la Universidad de Málaga, y financiado por la Fundación BBVA.

El proyecto Exhibitium debe enmarcarse como un estudio que pertenece a las Humanidades Digitales y a la investigación histórico-artística que utiliza métodos tecnológicos y computacionales para trabajar con grandes volúmenes de datos.

Definir las Humanidades Digitales es algo difícil, ya que requiere poner unos límites a algo tan amplio como son los estudios humanísticos y sociales, junto con el uso de la computación y el medio digital. Entendemos por Humanidades Digitales el conjunto de disciplinas humanísticas que utilizan métodos digitales y computacionales para producir nuevo conocimiento en cada uno de sus ámbitos de especialidad. Así, las Humanidades Digitales son una "transdisciplina" que relaciona los métodos digitales con los estudios de las ciencias sociales y humanas2.

Se puede decir, por tanto, que el proyecto Exhibitium, en el cual se lleva a cabo la

1. Exhibitium, http://exhibitium.com/ (Consultado: 06/06/2016).

2 El término Digital Humanities (Humanidades Digitales) ha sido definido en varios puntos mediante el manifiesto por unas Humanidades Digitales en el That Camp París. URSUA, N., "El pensar humanístico frente a las Humanidades Digitales", Límite. Revista Interdisciplinaria de filosofía y Psicología. Volumen 11, n³6, 2016, pp.33-34. 
indexación y el análisis de las exposiciones artísticas mediante tecnologías computacionales, supone un gran paso en la introducción de los estudios artísticos dentro del campo de las Humanidades Digitales, creando nuevas formas de investigación y de análisis de las exposiciones y obras artísticas.

Observando las investigaciones que realizan estudios culturales a través del análisis de datos, cabe nombrar a Lev Manovich, que ha acuñado el término de cultural analytics para referirse a "the analysis of massive cultural data sets and flows using computational and visualization techniques". Esto es lo que Franco Moretti designa como distant reading (lectura distante), frente al close reading (lectura cercana), característico, de las disciplinas humanísticas, el análisis en profundidad y en detalle de un conjunto de elementos pequeños como representativos de la totalidad. Moretti nos hace ver que ahora se puede llevar a cabo esa lectura distante, que permita conocer muchos más datos, que nos aleja, pero que proporciona otro tipo de conocimiento basado en la detección de patrones, tendencias o estructuras subyacentes.

En el ámbito internacional, merecen ser nombrados investigadores que estudian el arte y la cultura por medio de análisis computacionales como Maximilian Schich (miembro fundador del Edith O'Donnell Institute of Art History con el que realiza el proyecto Cultural Science Lab), Matthew Lincoln (investigador del Getty Research Institute) o Juan Luis Suárez (director de CulturePlex, en la Western University de Canadá).

\section{METODOLOGÍA}

A fin de poder realizar el análisis, el trabajo de campo ha consistido en la búsqueda de información sobre exposiciones artísticas celebradas en Cataluña, para poder indexar y registrar sus datos en la plataforma ExpoFinder (www.expofinder.es). Para ello, he analizado webs de museos, centros de arte y periódicos de Cataluña.

Tipos de fuentes:
a) Webs de museos y centros de arte
b) Prensa
c) Páginas webs de administraciones públicas como ayuntamientos, diputaciones, etc.


d) Webs de fundaciones y bancos

Tras la búsqueda de las exposiciones en las diversas webs de los museos y otras entidades, se ha descubierto que no existe una concordancia o unificación a la hora de utilizar una estructura de datos para la descripción y documentación de las exposiciones. Es apreciable que mientras algunas webs ofrecen bastantes datos, en otras son escasos y no siempre se ofrece el mismo tipo de información. Por ello, el proyecto Exhibitium ha propuesto la creación de un modelo de registro de datos, que es el que yo he utilizado para el desarrollo de mi proyecto.

El modelo de registro de datos contiene unos campos dependiendo del tipo de registro que se realice, ya sean Entidades, Personas, Publicaciones, Empresas o Exposiciones.

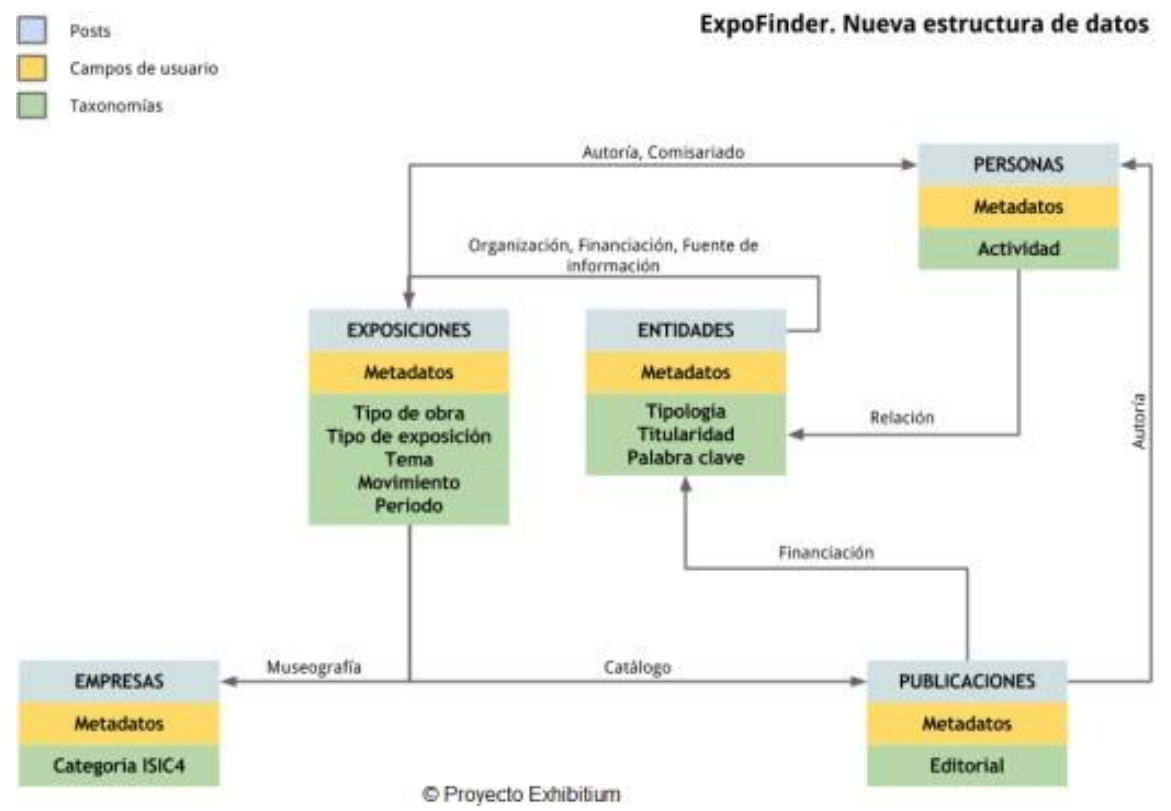

Respecto a la indexación de los datos, esta requiere de diversas fases. Primero se debe realizar la localización de la información en las diferentes webs de museos, centros de arte y otras webs de índole artístico. Tras ello, se realiza el proceso de datificación propiamente dicho, introduciendo en la plataforma ExpoFinder cada elemento de información de manera estructurada y descrita de acuerdo con las taxonomías registradas en vocabularios controlados específicos. Tras estos pasos, se procede a la limpieza, se examinan los datos para observar que no haya fallos y, por último, se realiza el análisis.

Al realizar la búsqueda de datos he hallado diversos problemas, ya que hayamos falta de datos, observando que algunas webs de museos o galerías no proporcionan información sobre el tipo de obra, el comisario, los artistas participantes en la exposición, las publicaciones, etc. 
En otras ocasiones algunas web han dejado de funcionar o no han incluido las exposiciones realizadas en años anteriores, o algunas entidades no presentan web y por tanto, de esta no se conoce ningún dato. A pesar de ello, el estudio ha sido amplio con un gran número de entidades y exposiciones, aunque se deba tener en cuenta que aquí no se hallan la totalidad de museos, centros de arte o galerías de toda la comunidad de Cataluña.

\begin{abstract}
ANÁLISIS
El análisis pretende desarrollar un conocimiento "tanto interpretativo-crítico como estratégicoprospectivo", para que pueda ser usado, utilizando la sistemática de análisis de redes, siguiendo la teoría Actor-Red3 de Bruno Latour. La teoría del Actor-Red es una sociología de las asociaciones, también llamado "teoría del actante-rizoma", basada en la explicación de lo social, como tipos de asociaciones transitorias que se caracteriza por la forma de reunirse y crear nuevas formas. Esta teoría conocida como TAR o ANT4 trata la relación de los actantes y la agencia (capacidad de provocar acciones y transformaciones), de modo que los fenómenos socio-culturales se entienden como el conjunto de relaciones establecidas entre actores heterogéneos que configuran redes cambiantes, más o menos complejas.

El corpus sobre el que se ha realizado el análisis de las exposiciones en Cataluña tiene un tamaño de 72 entidades, 473 exposiciones y 466 personas (artistas y comisarios). Las cuestiones que he tratado de resolver son:

1. ¿Cuál es la densidad expositiva en Cataluña? ¿Cuál es la densidad expositiva por ciudad?

En la provincia de Barcelona, de 2010 a 2015, de acuerdo con el corpus de datos registrados en ExpoFinder se realizaron en total 124 exposiciones, de las cuales 106 se realizaron en Barcelona y el resto en las distintas localidades. Analizando los números, podemos ver que desde 2010 a la actualidad, el número de exposiciones realizadas por año ha ido aumentando progresivamente.
\end{abstract}


9 Proyecto Exhibitium

Cuenta vs Region por Localidad

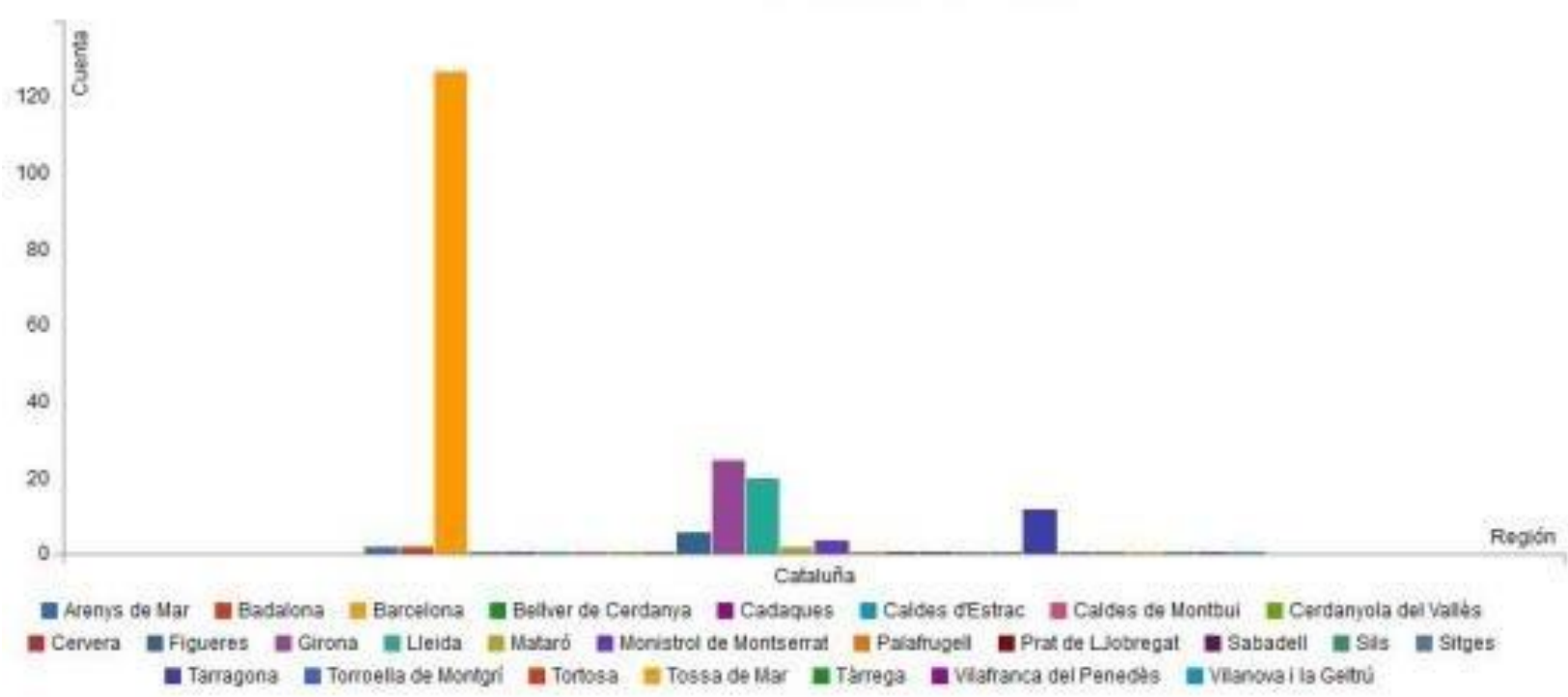

2. ¿Con qué frecuencia cambian de exposición cada museo o entidad a lo largo de un año?

El museo que ha realizado más exposiciones entre 2010 y 2015 ha sido el MACBA, que fue el que cambió más de exposición por año, ya que en 2010 realizó 10 exposiciones; en 2011 fueron 11; en 2012, 19; en 2013, 5; en 2014, 17 y en 2015 realizó 11.

Tras este museo, el que realizó más exposiciones fue el Centre d' Art la Panera con un total de 72 exposiciones en los cinco años, realizando en 2010 más exposiciones que el MACBA en ese mismo año, pues llevaron a cabo 18 exposiciones. En 2011, se hicieron 14 exposiciones; en 2012, 11, en 2013, 8; en 2014, 12 y en 2015 fueron 9. 


\section{Porcentaje de exposiciones realizadas por entidad entre 2010 y 2015}

Víctor Lope Arte Contemporáneo Universitat Autònoma de Barcelona Teatre-Museu Dal Photographic Social Vision Obra Sodial "la Caixa"

Museu Picasso

Museu Nacional Arqueològic de Tarragona Museu Marès de la Punta

Museu Egipci de Barcelona Museu del Ferrocarril de Catalunya Museu del Cinema. Col-lecció Tomàs Mallol Museu de Montserrat Museu de la Música Museu de l'Empordà

Museu de Badalona

Museu d'història Dels Jueus Museu d'art Modem de la Diputació de Tarragona Museu d'art de Cerdanyola (MAC) - Can Domènech Museu d'arqueologia de Catalunya

Museu Comarcal de Cervera

Museo Municipal de Cadaqués Museo del Gas

Michael Dunev Art Projects L'Associació d'Artistes Plàstics i Visuals del.. Institut de Biotecnologia i de Biomedicina Vicent.. Galería Trama Galería Miguel Marcos Galería Marlborough Barcelona Galería Ignacio de Lassaletta Galeria Sicart

Galeria Balaguer Fundación La Caixa Fundación Banco Sabadell Fundación Abertis Fundació Ramón Calsina Fundació Joan Miró Fundació Catalunya La Pedrera Fundació Albertis Fujifilm

Festival Internacional de Mèxic a Barcelona El Quatre Diputació de Ueida Diputació de Barcelona Consulado General de México en Barcelona Cervezas Moritz Centre de Cultura Contemporània de Barcelona Casa Salvador Dalí - Portlligat CaixaBank Arxiu Nacional de Catalunya Amical Wikimedia Ajuntament de Girona Adn Galería

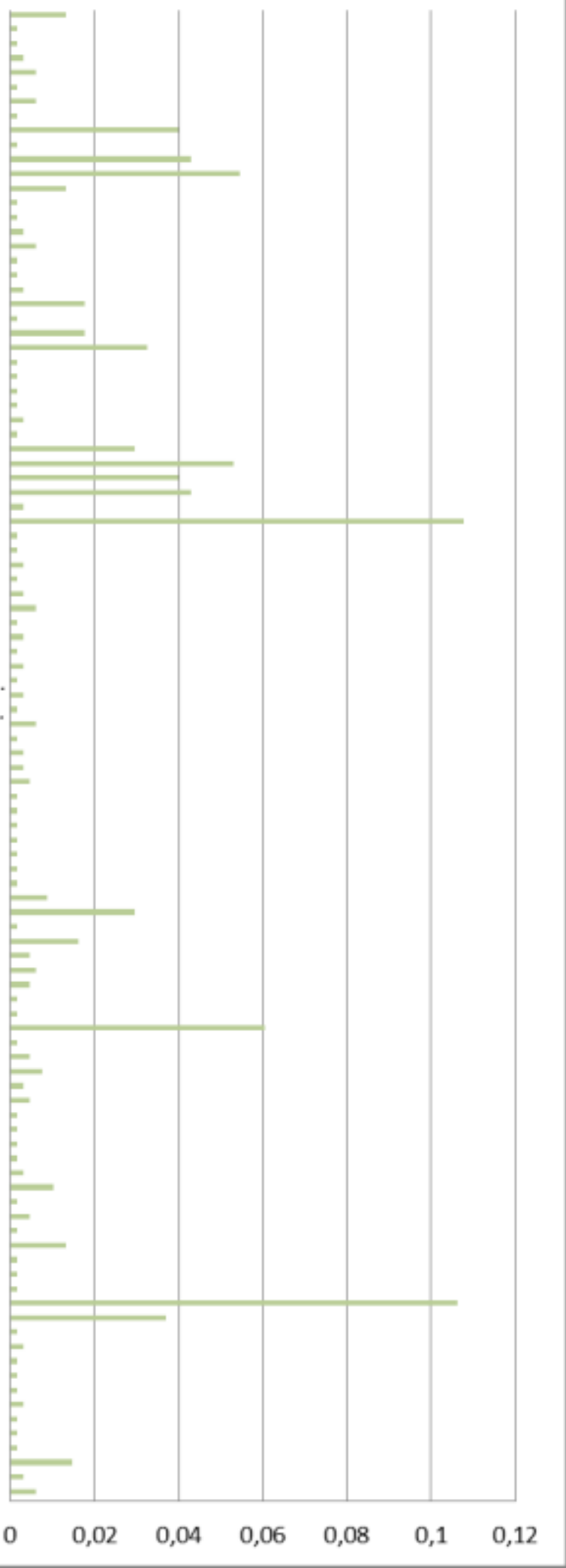


3. ¿Qué tipo de obra se está exhibiendo más en las exposiciones?

Entre los años 2010 y 2015, se hace visible que el gran número se localiza bajo el término "artes visuales". Dentro de estas, vemos que es "pintura" el tipo de obra más relevante, en detrimento de otras propuestas.

Tras la pintura, el objeto más expuesto es la "fotografía". Después de la pintura y la fotografía, se puede apreciar que otro tipo de obra bastante exhibido pertenece al "media art".

Es destacable apreciar la diversidad tipológica de obras que se exhiben en las exposiciones de grandes ciudades, como Barcelona, con 65 tipos de obras, Gerona, con 36 tipos distintos, Lleida, con 53 tipos diferentes y en Tarragona, con 33.

4. ¿Qué artistas coinciden en más de una exposición? ¿Y en qué exposición coaparecen?

Tras la realización del análisis, cabe expresar que en la base de datos hallamos 473 exposiciones con 8.984 autores, de las cuales, las exposiciones colectivas (co-ocurren dos autores o más) son 138.

El análisis se realiza a través de las relaciones de co-ocurrencias. El análisis de ocurrencias se basa en el número de palabras asociadas, partiendo de descriptores o palabras clave, y tomando como punto de partida una matriz de datos (documentos por palabras clave) que generan la matriz de ocurrencias5 . De esta forma, dos palabras, en este caso artistas, coocurren cuando aparecen paralelamente en el mismo documento, en este caso en la misma exposición.

Los grafos muestran en los vértices los actores en este caso, artistas que coinciden y que se relacionan a través de redes, arcos o enlaces, que forman la estructura del actor-red. De esta forma, observamos en los diferentes grafos las uniones del conjunto de vértices que presentan los actores y las formas de unirse entre ellos.

Entre los artistas que aparecen o coocurren en más exposiciones podemos destacar a Pablo Ruiz Picasso, Salvador Dalí, Antoni Tàpies i Puig, Joan Miró, Francisco de Goya y Lucientes, Ignasi Aballí, entre otros.

5 RUIZ-BAÑOS R. y BAILÓN-MORENO, R., "El método de las palabras asociadas (II): Los ciclos de vida de los temas de investigación" 
Podemos observar en el grafo la relación de Picasso con Ramón Casas, con Fernand Léger y con Isidre Nonell. También es destacable la gran relación que aparece entre Ramón Casas e Isidre Nonell, que se muestra con una red intensa. Por otro lado, menor es la relación que tiene Picasso con Roberto Matta Echaurren y con Ricard Canals.

Analizando las redes de la artista contemporánea Julia Montilla, nos encontramos con un grafo más complejo que el observado con Picasso. La red de Julia es distribuida, frente a la de Picasso que es más centralizada. Vemos una interrelación de diversos artistas contemporáneos entre unos y otros, situando en el centro a Julia Montilla con una fuerte relación con Dora García, Jordi Mitjá, Ignacio Uriarte, Daniel Canogar, Abigail Lazkoz, Ignasi Aballí, Antonio Ortega, Helena Almeida, David Bestué, Alicia Framis, Carles Congost, Martí Anson y Luz Broto. Y diversas relaciones entre artistas, hallando redes distribuidas de unos hacia otros.

En el grafo de los artistas se puede apreciar que el MACBA y la Fundación Joan Miró se encuentran en la zona céntrica representada con color verde y con distintas redes a varios artistas y comisarios. Otras redes son las de los artistas y comisarios en violeta, naranja y rojo.

5. ¿Qué artistas son catalanes? ¿Hay mayoría de artistas catalanes en las exposiciones o son de diferentes comunidades o países?

Los principales artistas sobre los que se han desarrollado más exposiciones son catalanes o han pasado parte de su vida allí. Así, vemos a Dalí o Andrea Michaelsson Btoy.

Otros artistas participantes en exposiciones en Cataluña son españoles. Vemos también artistas de países como Estados Unidos, Francia, Holanda. 


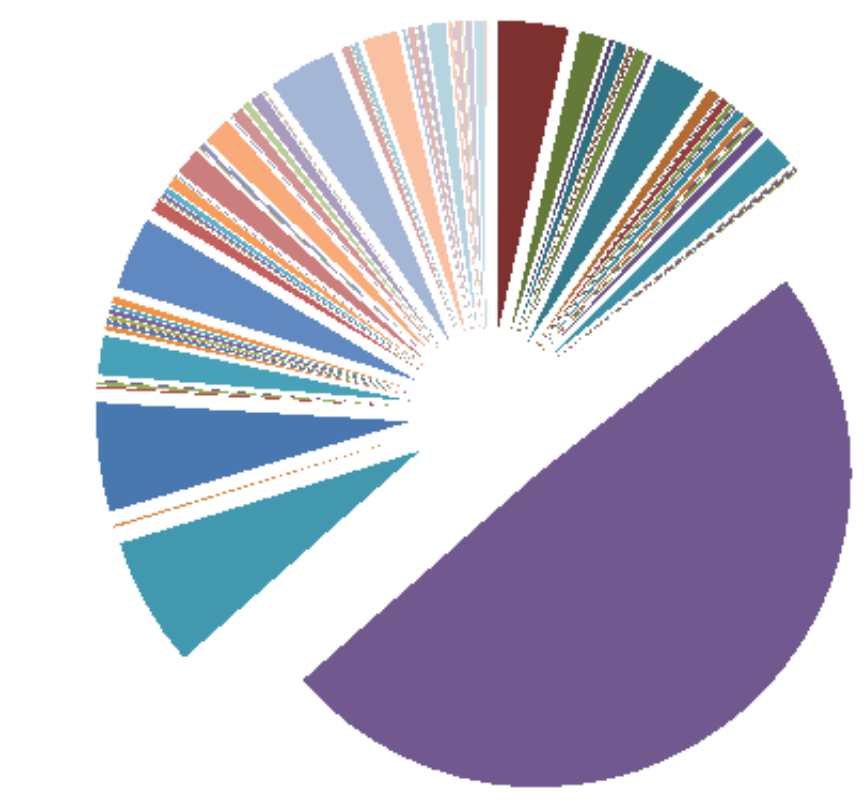

- Albania

alemania

I argentina

- Australia

austria

Benín

- Bielorrusia

- Bosnia-Hercegovina

Brasil

- Bulgaria

Belgica

Un canadá

- Checoslovaquía

Chile

nchina

- Chipre

Corea delS

Corea del Sur

- Costa de Martil

- Costa Rica

- Croacia

- Desconocido

- Dinamarca

- Egipto

- El Salvador

Eslovaquia

- España

- Estados Unidos

- Finlandia

- Francia

- Gran Bretaña

- Grecia

- Guatemala

- Holanda

- Hungría

= India

urak

- Irlanda

- Irán

- Islandia

- Israel

- Italia

- Japón

Kenia

- Lituania

- Líbano

Marruecos

- Montenegro

- México

Nigeria

- Noruega

- NuevaZelanda

null

- Pakistán

Paḱs

Peru

Polonía

- Portugal

- Praga

- Puerto Ríco

arino Unido

- República Checa

Eepública Democrática del Congo

- República Dominicana

- Rumania

Rusia

Serbia

" Sudáfrica

Eudán

insuecia

Suiza

$\checkmark$ Tailandia

Territorios Palestinos

Nacionalidades de artistas y comisario

- Turquía

- Ucrani

- Uruguay

Venezuela
Vietnan

6. ¿Cuáles son los artistas que han tenido más presencia en las exposiciones?

El artista al que se le han dedicado más exposiciones ha sido Pablo Picasso, con un total de 6 exposiciones entre 2010 y 2015. Tras él, hallamos a Salvador Dalí con 4 exposiciones entre los 
cinco años. Tras estos hallamos a Andrea Michaelsson Btoy con 3 exposiciones.

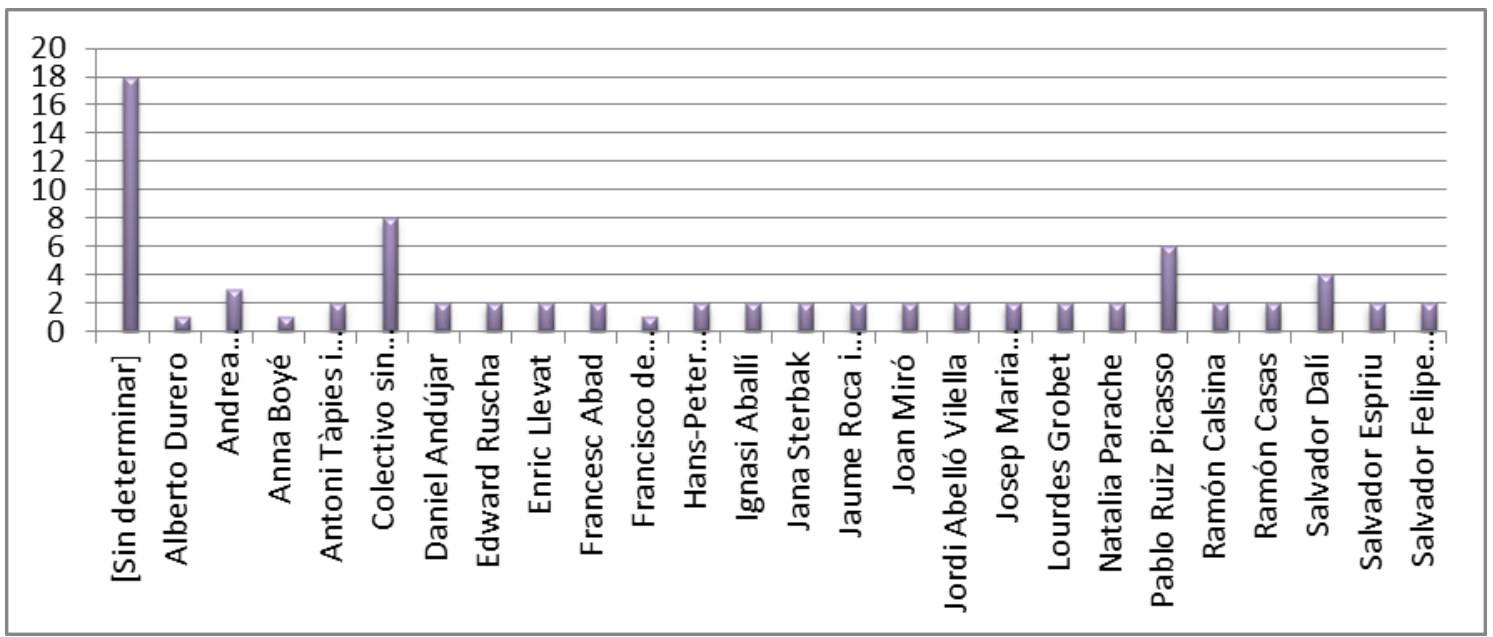

7. ¿Cuáles son los periodos sobre los que se han realizado más exposiciones?

El mayor número de exposiciones se ha ocupado del arte del siglo XXI y el siglo XX. Tras estos periodos, hallamos que se han dedicado un mayor número de exposiciones al siglo XIX y al XVIII.

8. ¿Cuáles han sido los movimientos/poéticas que han ocupado más exposiciones?

El arte contemporáneo ocupa el principal número de exposiciones. Tras este, aparece el modernismo, realizándose muchas de las exposiciones sobre el modernismo catalán. Posteriormente son las vanguardias sobre las que se producen más exposiciones. El surrealismo continúa detrás de estos, con la producción de 17 exposiciones en toda Cataluña entre 2010 y 2015.

Después localizamos el arte emergente, sobre el cual se han realizado 16 exposiciones, 13 han sido las exposiciones realizadas sobre arte conceptual. Y 10 exposiciones sobre arte abstracto, otras 10, sobre el Barroco, otras 10 sobre el Impresionismo y otras 10 sobre el Romanticismo.

9. ¿Qué comisarios encontramos? ¿Con qué entidades se relacionan?

Los profesionales que han comisariado más exposiciones son Montse Aguer con 5 exposiciones, Arnau Puig con 3 exposiciones, Ingrid Guardiola con otras 3, Moritz Küng con 3 y Teresa Blanch con tres también. Otros comisarios que han organizado al menos dos exposiciones ente 2010 y 2015 son Anna Capella, Brigitte Léal, Carles Guerra, Carolina López, 
Elvira D’Amicone, Eva Jove, Francesc Muñoz, Joan Miquel Llodrà i Nogueras, Jorge Ribalta, Josep Canals, Luis Manuel Gonzálvez, Manuel Guerrero, Mercè Doñate, Nuxu Perpinyà Salvatella, Rocío Santa Cruz, Rosa María Gil, Toni Coromina y Valentín Roma. Montse Aguer ha comisariado exposiciones para la Fundación Gala-Salvador Dalí y para el Teatro-Museo Dalí. Ingrid Guardiola ha llevado a cabo exposiciones para el Departamento de Cultura de la Generalitat de Cataluña, la Diputación de Gerona y el Museo del Cinema, Colección Tomàs Mallol.

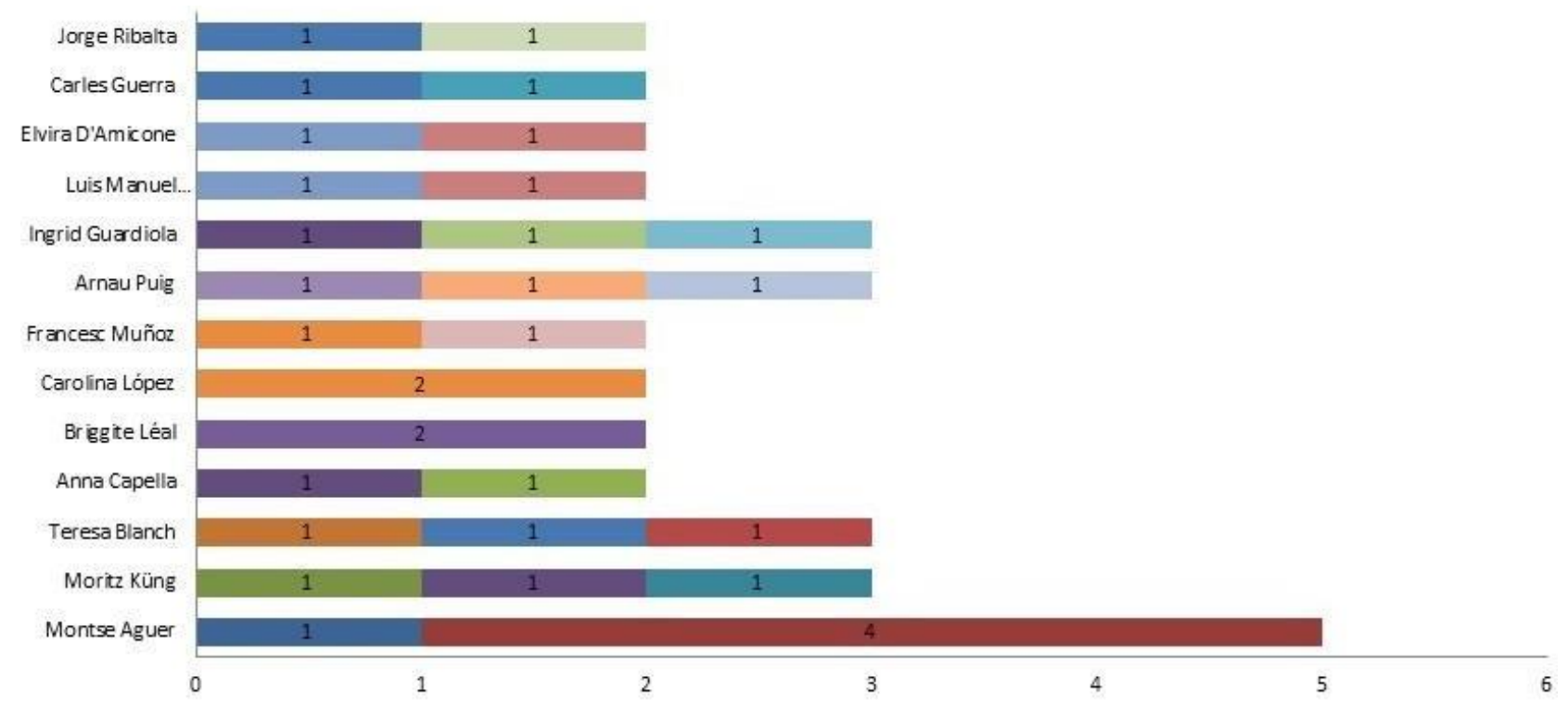

\begin{tabular}{|c|c|c|c|}
\hline - Fundación Gala-Salvador Dali & - Teatro-Museo Dalí & = Centro de Arte Sta Mónica & " Genralidad de Cataluña \\
\hline - Raiña Lupa & Ealería Toni Tapies & MACBA & Museo de Montser rat \\
\hline = Museo de L'Empordà & w Fundación Miró & = Fundación Antoni Tàpies & $=C C C B$ \\
\hline Eundación Arqueológica Clos & Museo Egipcio de Barcelona & Diputación de Gerona & Museo Marítimo de Barcelona \\
\hline Museo del Cine. Tomàs Mallol & Galería Mayoral & Fundación Cuixart & Diputación de Barcelona \\
\hline
\end{tabular}

Artistas que han comisariado dos o más exposiciones

10. ¿Qué entidades realizan exposiciones con más comisarios, es decir, por cuál entidad han pasado el mayor número de comisarios?

El Museo d' Art Contemporani de Barcelona es la entidad que ha llevado a cabo exposiciones con un mayor número de comisarios, con un total de 8. La Fundació Joan Miró, el Museo d'historia de la Ciutat (Girona) y el Museu Picasso fueron los que contaron con 7 comisarios cada uno para las exposiciones entre 2010 y 2015. Y otras entidades como el Centre de Cultura 
Contemporània de Barcelona y el Museu d' Art Jaume Morera en los cuales participaron 6 comisarios distintos en cada uno. El ajuntament de Girona, el Centre d' Art la Panera y el departamento de cultura de la Generalitat de Catalunya contaron con 5 comisarios cada uno. Y otras entidades que realizaron exhibiciones con 4 comisarios fueron: Fundació Antoni Tàpies, Fundación La Caixa, Museu d'art Modern de la Diputació de Tarragona, Museu d' historia dels Jueus, Museu de Montserrat y la Obra Social "La Caixa".

11. ¿Qué instituciones organizan más exposiciones?

El Centro Cultural Contemporáneo de Barcelona organizó 6; también, el Centro d' Art la Panera organizó 6; y la Fundación Joan Miró, 6. El porcentaje de exposiciones que realizaron estos es del 3'6\% sobre 100 del total de exposiciones. Otras instituciones que organizaron 6 exposiciones entre 2010 y 2015 fueron el Museu d' Art Contemporani de Barcelona; el Museu d' Art Jaume Morera; el Museu d' Art Modern de la Diputació de Tarragona; el Museu d' historia de la ciutat de Gerona; el Museu d' historia del Jels Jueus; el Museo de Lleida. Diocesa I Comarcal; el Museo del Cinema. Colleció Tomàs Mallol, el Museu Nacional d' Art de Catalunya, el Museu Picasso, El Departamento de Cultura de la Generalitat de Catalunya organizó 5 exposiciones; La Fundación La Caixa realizó 5; el Museu Nacional Arqueològic de Tarragona organizó 5; la Obra Social la Caixa organizó 5. Estos ocuparon un porcentaje del 2’55\% cada uno, y el Ajuntament de Girona organizó 4, presentando un porcentaje del 2,04\%. El resto de instituciones realizaron 3, 2 o 1 sola exposición. Así, los que realizaron 3 exposiciones ocuparon un 1'53\%, las de 2 un 1'02\% y las de una sola exposición ocupan un $0{ }^{\prime} 51 \%$ del total.

Observando el gráfico general y el detalle del grafo de España, destacan la Fundación Antoni Tàpies, la Fundación La Caixa, El Departamento de Cultura de la Generalitat de Cataluña, CaixaForum, la Fundación Joan Miro y el MACBA con una red centralizada, cercano en el ámbito nacional al Museo Centro de Arte Reina Sofía.

12. ¿Cuál es la nacionalidad de los artistas y comisarios? ¿Cuál es el porcentaje de género en los artistas y comisarios participantes en las exposiciones artísticas?

Hallamos 804 artistas españoles frente a 184 comisarios españoles. 59 artistas y un comisario alemán, 22 artistas argentinos. También observamos 43 artistas belgas y un solo comisario belga, 10 artistas canadienses y ningún comisario de esa nacionalidad, 110 artistas 
estadounidenses y 8 comisarios. Encontramos 92 artistas franceses y 11 comisarios. Tenemos 61 artistas italianos y 2 comisarios; de Reino Unido hay 57 artistas y 6 comisarios,33 artistas holandeses, 30 artistas rusos; 23 artistas de México y 2 comisarios; 16 artistas de Suiza; 11 artistas japoneses; 11 artistas portugueses y un comisario. Hay 331 artistas mujeres frente a los 1217 artistas hombres. Y hallamos 84 comisarias frente a 142 comisarios.

Se puede ver en el gráfico los artistas y comisarios hombres se muestran en color violeta y las mujeres se presentan en naranja, y el azul representa los grupos colectivos.

\section{CONCLUSIONES}

Con los datos obtenidos del análisis del proyecto, he podido realizar preguntas interesantes para la Historia del Arte (¿Cuál es la densidad expositiva en Cataluña? ¿con qué frecuencia cambian de exposición cada museo o entidad a lo largo de un año? ¿qué tipo de obra se está exhibiendo más en las exposiciones? ¿qué artistas coinciden en más de una exposición? ¿en qué exposición coaparecen? ¿encontramos más artistas reconocidos en lugar de artistas de menos renombre? ¿cuáles son los periodos sobre los que se han realizado más exposiciones?, ¿cuáles han sido los movimientos/poéticas que han ocupado más exposiciones? ¿qué instituciones organizan más exposiciones? ¿cuál es la nacionalidad de los artistas y comisarios? ¿cuál es el porcentaje de género en los artistas y comisarios participantes en las exposiciones artísticas? entre otras).

Con la búsqueda de las exposiciones en las diversas webs de entidades que desarrollan exposiciones artísticas, he podido comprobar la falta de unificación entre estas respecto a los datos facilitados en sus páginas. De esta forma, cabe destacar que, aunque los museos o centros de arte son autónomos, debería existir algún tipo de normativa común a la hora de facilitar los datos, y debería de promoverse la búsqueda de los datos abiertos, a fin de facilitar la generación de nuevo conocimiento.

En los análisis he observado la cantidad de exposiciones en las que participan artistas catalanes. También es apreciable que un gran número de exposiciones se dedique al modernismo catalán, como movimiento destacado en la historia del arte en España y sobre todo en Cataluña. Otros análisis respecto a los artistas han dado a conocer el número de exposiciones que se les han dedicado a artistas reconocidos como Picasso o Dalí y las redes que hallamos de estos y otros artistas, observando que a Picasso se le han dedicado el mayor número de exposiciones, pero a la hora de interactuar, la relación con otros artistas por la producción de 
exposiciones colectivas, es menor que la red que se puede ver en artistas más contemporáneos.

Tras el estudio se puede corroborar que generalmente los grandes centros son los que producen más exposiciones, como el MACBA. Tras él, aparece el Centre d' Art la Panera de Lérida con una gran densidad expositiva, realizan en 2010 hasta 18 exposiciones. Vemos, así, que no solo hay centros con una gran oferta expositiva en la capitalidad de Barcelona, sino también en otras ciudades. Las grandes instituciones cuentan con un mayor número de comisarios para las exposiciones, como es el caso del MACBA, y los centros más pequeños suelen contar con menos comisarios con los que llevan a cabo más de una exposición. Respecto a los tipos de obras expuestas, sigue predominando la pintura y la fotografía.

Es destacable apreciar que en las exposiciones el porcentaje de mujeres artistas sigue siendo reducido, pero encontramos más mujeres en el puesto de comisarias que en el de artistas, deduciendo un aumento del papel de la mujer en las exposiciones, dejando de ser meras musas de los artistas. Además, hallamos también artistas y comisarios de géneros masculino y femenino de diversas nacionalidades, siendo mayor la participación de españoles y también de estadounidenses, franceses, italianos, alemanes e ingleses. Vemos un 56\% de hombres comisarios y $34 \%$ de comisarias, el porcentaje de los artistas hombres es del $77^{\prime} 82 \%$, y de las artistas del $15,62 \%$. Y respecto a la nacionalidad, tienen la española el $49 \%$ de las personas participantes en las exposiciones.

Por último, se puede observar que se han llevado a cabo más exposiciones sobre el siglo XIX y XX, dándosele mayor prioridad a estos periodos y hallando movimientos destacados a los que se les dedican más exposiciones como el modernismo, las vanguardias y el surrealismo.

\section{REFERENCIAS BIBLIOGRÁFICAS}

BENTKOWSKA-KAFEL, A., "Debating Digital Art History", International Journal for Digital Art History, $n^{\circ}$ 1, 2015, pp.50-65

DRUCKER, J., Doing Art History Digitally/ Doing Digital Art history? , http://digitalarthistory.weebly.com/uploads/6/9/4/3/6943163/johannadrucker_remarks_gettyd ah- lab_2013.pdf (Consultado: 11/09/2016).

LATOUR, B., Reassemblig the Social: An Introduction to Actor-Network-Theory, Oxford, Edit.

Oxford, 2007. 
MANOVICH, L., The Science of Culture? Social Computing, Digital Humanities and Cultural Analytics, http://manovich.net/index.php/projects/cultural-analytics-social-computing (Consultado 24/08/2016).

RAMÍREZ-SÁNCHEZ, M., "Las Ciencias y Técnicas Historiográficas en el contexto de las Humanidades Digitales: Oportunidades para su Desarrollo. Universidad de Las Palmas de Gran Canaria”, Alicia Marchant Rivera y Lorena Barco Cebrián (eds.), 'Dicebamus hesterna die...' Estudios en homenaje a los profesores Pedro J. Arroyal Espigares y $M^{a}$ Teresa Martín Palma, Universidad de Málaga, Málaga, 2016, pp.1-7.

RODRÍGUEZ ORTEGA, N. y TAÍN GUZMÁN, M., Teoría y Literatura artística en España. Revisión historiográfica y estudios contemporáneos. Real Academia de San Fernando, Madrid, 2015. pp. 22-23.

RODRÍGUEZ ORTEGA, N., y TENOR POLO, C., "Modelo de datos y estructura de metadatos para el registro de exposiciones artísticas temporales", http://exhibitium.com/wpcontent/uploads/2016/05/guia-datos-metadatos.pdf (Consultado 20/06/2016).

RUIZ-BAÑOS R. y BAILÓN-MORENO, R., "El método de las palabras asociadas (II): Los ciclos de vida de los temas de investigación" en Boletín de la Asociación Andaluza de Bibliotecarios, nº 54, Enero-99, Málaga, pp. 59-71.

URSUA, N., "El pensar humanístico frente a las "Humanidades Digitales"”, Límite. Revista Interdisciplinaria de filosofía y Psicología. Volumen 11, №36, 2016.

Cultural Science Lab, https://www.utdallas.edu/arthistory/cultural-science/ (Consultado: 12/07/2016).

CulturePlex. Juan Luis Suárez, http://www.cultureplex.ca/team/juan-luis-suarez/ (Consultado 12/07/2016).

Culturomics, http://www.culturomics.org/(Consultado: 25/08/2016).

Dr. Phil. Maximilian Schich, http://www.schich.info/ (Consultado: 12/07/2016). Exhibitium, http://exhibitium.com/ (Consultado: 06/06/2016).

"Fundamentosde Ciencimetría de redes" en Redes 2005, http://www.ugr.es/ bailonm/redes/ayuda/index.htm (Consultado: 17/07/2016).

Getty Research Institute (GRI), https://www.getty.edu/research/(Consultado: 12/07/2016). Matthew Lincoln, PhD, http://matthewlincoln.net/ (Consultado: 12/07/2016).

The Edith O' Donnell Institute of Art History, https://www.utdallas.edu/arthistory/ (Consultado: 12/07/2016). 